\title{
A CANONICAL FORM FOR SYMMETRIC AND SKEW-SYMMETRIC EXTENDED SYMPLECTIC MODULAR MATRICES WITH APPLICATIONS TO RIEMANN SURFACE THEORY
}

\author{
BY \\ ROBERT ZARROW(')
}

ABSTRACT. The (extended) symplectic modular group $\left(\Lambda_{n}\right) I_{n}$ is the set of all $2 n \times 2 n$ integer matrices $M$ such that

$$
\left(M J^{t} M= \pm J\right), \quad M J^{t} M=J, \quad J=\left[\begin{array}{cc}
0 & I \\
-I & 0
\end{array}\right],
$$

$I$ being the $n \times n$ identity matrix. Let $S_{n}=\left\{M \in \Lambda_{n}-I_{n} \mid M=-{ }^{t} M\right\}$ and $T_{n}=\left\{M \in \Lambda_{n}-V_{n} \mid M={ }^{t} M\right\}$. We say $M \sim N$ if there exists $K \in \Gamma_{n}$ such that $M=K N^{t} K$. This defines an equivalence relation on each of these sets separately and we obtain a canonical form for this equivalence.

We use this canonical form to study two types of Riemann surfaces which are conformally equivalent to their conjugates and obtain characterizations of their period matrices. We also obtain characterizations of the symplectic matrices which the conformal equivalence induces on the first homology group. One type of surface dealt with is the symmetric Riemann surfaces, i.e. those surfaces which have a conjugate holomorphic self-map of order 2. The other type of surface studied we we call pseudo-symmetric surfaces. These are the hyperelliptic surfaces with the property that the sheet interchange is the square of a conjugate holomorphic automorphism.

1. Introduction. In this paper we will solve a problem in number theory that arises in the study of compact Riemann surfaces which have a conjugate holomorphic homeomorphism (see $\S 3$ ). The problem is one of finding a canonical form for a set of matrices on which an equivalence relation is defined.

The symplectic modular group $\Gamma_{n}$ is defined to be the set of all $2 n \times 2 n$ integer matrices $K$ such that

$$
K J_{n}{ }^{t} K=J_{n}
$$

Received by the editors November 13, 1973.

AMS (MOS) subject classifications (1970). Primary 15A21, 15A36, 10M20, 32G20, 57A05: Secondary 15A63, 10E25.

(1) The results in this article are contained in the author's Ph. D. thesis written at Cornell University under the direction of Professor Clifford J. Earle. The author would like to thank him as well as Professor Alex Rosenberg and the referee for several useful suggestions. 
where

$$
J_{n}=\left[\begin{array}{cc}
0 & I_{n} \\
-I_{n} & 0
\end{array}\right]
$$

and $I_{n}$ is the $n \times n$ identity. The extended symplectic modular group $\Lambda_{n}$ is the set of all $2 n \times 2 n$ matrices $K$ such that

$$
K J_{n}{ }^{t} K= \pm J_{n} \text {. }
$$

If we write $K=\left[\begin{array}{ll}Q & R \\ T\end{array}\right]$, where $Q, R, S$ and $T$ are $n \times n$ integer matrices, then it is easy to see $K \in \Gamma_{n}$ iff

$$
Q^{t} T-R^{t} S=I_{n}, \quad Q^{t} R=R^{t} Q, \quad S^{t} T=T^{t} S .
$$

Also $K \in \Lambda_{n}$ iff

$$
Q^{t} T-R^{t} S= \pm I_{n}, \quad Q^{t} R=R^{t} Q, \quad S^{t} T=T^{t} S .
$$

If $K \in \Gamma_{n}$, then

$$
K^{-1}=\left[\begin{array}{cc}
{ }^{t} T & -{ }^{t} R \\
-{ }^{t} S & { }^{t} Q
\end{array}\right]
$$

and if $K \in \Lambda_{n}-\Gamma_{n}$, then

$$
K^{-1}=\left[\begin{array}{cc}
-{ }^{t} T & { }^{t} R \\
{ }^{t} S & -{ }^{t} Q
\end{array}\right]
$$

Furthermore, $\left[\Lambda_{n}: \Gamma_{n}\right]=2$.

Now let

$$
S_{n}=\left\{M \in \Lambda_{n}-\Gamma_{n} \mid M=-{ }^{t} M\right\}, \quad T_{n}=\left\{M \in \Lambda_{n}-\Gamma_{n} \mid M={ }^{t} M\right\} .
$$

A simple calculation shows that if $A, B$, and $C$ are $n \times n$ integer matrices, then the elements of $S_{n}$ are precisely those matrices of the form

$$
\left[\begin{array}{cc}
B & A \\
-{ }^{t} A & C
\end{array}\right]
$$

where $B=-{ }^{t} B, C=-{ }^{t} C, A B=-B^{t} A, C A=-{ }^{t} A C$ and $A^{2}-B C=-I_{n}$. Similarly the elements of $T_{n}$ are precisely those matrices of the form

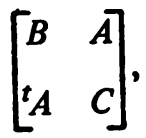


where $B={ }^{t} B, C={ }^{t} C, A B=B{ }^{t} A, C A={ }^{t} A C$ and $A^{2}-B C=I_{n}$.

Now we say $M \sim N$ iff there exists $K \in \Gamma_{n}$ such that $M=K N^{t} K$. It is easy to see that this is an equivalence relation on each of these sets separately. The following two theorems give a canonical form for this equivalence.

THEOREM A. (1) If $n$ is odd, then $S_{n}$ is empty.

(2) If $n$ is even, then every element of $S_{n}$ is equivalent to

$$
\left[\begin{array}{cc}
J_{k} & 0 \\
0 & -J_{k}
\end{array}\right],
$$

where $n=2 k$.

THEOREM B. Every element of $T_{n}$ is equivalent to exactly one of the matrices

$$
M(r, t)=\left[\begin{array}{cc|cc}
I_{r} & 0 & I_{s} & 0 \\
0 & 0 & 0 & F_{t} \\
\hline I_{s} & 0 & 0 \\
0 & F_{t} & 0
\end{array}\right],
$$

where $r=0,1,2, r \leqslant s, s+2 t=n$,

$$
F_{1}=\left[\begin{array}{ll}
0 & 1 \\
1 & 0
\end{array}\right]
$$

and

$$
F_{t}=\left[\begin{array}{lll}
F_{1} & & \\
& F_{1} & \\
& & \\
& & F_{1}
\end{array}\right], \quad 2 t \times 2 t .
$$

REMARK 1.1. The notation introduced in this section will be used throughout the entire paper. We add the following. If $S$ is a symmetric $n \times n$ integer matrix then

$$
G(S)=\left[\begin{array}{ll}
I_{n} & S \\
0 & I_{n}
\end{array}\right] .
$$

If $U$ is an $n \times n$ unimodular matrix, then 


$$
H(U)=\left[\begin{array}{cc}
U & 0 \\
0 & { }^{t} U^{-1}
\end{array}\right] .
$$

It is clear that both $G(S)$ and $H(U)$ are in $\Gamma_{n}$. We also use a direct sum notation for symplectic matrices. If

$$
M_{i}=\left[\begin{array}{ll}
A_{i} & B_{i} \\
C_{i} & D_{i}
\end{array}\right] \in \Lambda_{n_{i}}\left(\Gamma_{n_{i}}\right),
$$

$i=1,2$, then we denote

$$
M_{1}+M_{2}=\left[\begin{array}{cc|cc}
A_{1} & 0 & B_{1} & 0 \\
0 & A_{2} & 0 & B_{2} \\
\hline C_{1} & 0 & D_{1} & 0 \\
0 & C_{2} & 0 & D_{2}
\end{array}\right] .
$$

It is easy to check that $M_{1}+M_{2} \in \Lambda_{m}\left(\Gamma_{m}\right)$, where $m=n_{1}+n_{2}$.

All numbers, matrices and vectors dealt with in this paper may be assumed to be integers or have integer entries unless it is stated otherwise. Also subscripts which indicate the size of a matrix (e.g., " $n$ " in " $I_{n}$ ") are occasionally omitted when it is clear what the size is. By the expression "apply $K$ to $M$ " we mean form the product $K M^{t} K$.

REMARK 1.2. This problem was suggested by a situation in the theory of compact Riemann surfaces.

Let

$$
S_{n}=\left\{F \in \Lambda_{n}-\Gamma_{n} \mid F^{2}=-I_{2 n}\right\}, \quad T_{n}=\left\{F \in \Lambda_{n}-\Gamma_{n} \mid F^{2}=I_{2 n}\right\} .
$$

If $X$ is a compact Riemann surface of genus $n$ which has a conjugate holomorphic homeomorphism of order 2 , then the induced action on $H_{1}(X)$ with respect to a canonical homology basis is a matrix in $T_{n}$. If $n$ is even, then it is shown in [2] that there exist hyperelliptic surfaces with the property that the sheet interchange is the square of a conjugate holomorphic homeomorphism. In this case the induced action on $H_{1}(X)$ with respect to a canonical homology basis is represented by an element of $S_{n}$. It is natural to ask if every element of $S_{n}$ and of $T_{n}$ arise in this way. An affirmative answer is given in $\S 3$.

In order to obtain a normalization of the period matrices of these surfaces we consider the equivalence $E \sim F$ iff there exists a $K \in \Gamma_{n}$ such that $E=$ $K F K^{-1}$. This is an equivalence relation on $S_{n}$ and $T_{n}$ separately. Furthermore, if 


$$
l(E)=\left[\begin{array}{cc}
I & 0 \\
0 & -I
\end{array}\right] E\left[\begin{array}{cc}
0 & I \\
I & 0
\end{array}\right],
$$

then $E \in S_{n}$ (resp. $\left.T_{n}\right)$ iff $l(E) \in S_{n}$ (resp. $T_{n}$ ) and $E \sim F$ iff $l(E) \sim l(F)$.

Theorems A and B may thus be restated in the following form.

THEOREM A'. The same statement as Theorem A with " $S_{n}$ " replaced by " $S_{n}$ ".

THEOREM $\mathrm{B}^{\prime}$. Every element of $T_{n}$ is equivalent to exactly one of the matrices

$$
Y(r, t)=\left[\begin{array}{cc|cc}
I_{s} & 0 & I_{r} & 0 \\
0 & F_{t} & 0 & 0 \\
\hline 0 & -I_{s} & 0 \\
0 & 0 & -F_{t}
\end{array}\right] .
$$

The following corollary is also useful.

COROllary 1.3. If $M \in S_{n} \cup T_{n}$ then $M \sim-M$.

A proof of this is given at the end of $\S 2$.

REMARK 1.4. It should be said that Theorems $A^{\prime}$ and $B^{\prime}$ give descriptions of certain cohomology sets. There is a nonabelian cohomology theory (e.g., [5]) which we describe here. Let $G$ and $A$ be topological groups, $A$ having the discrete topology and let $G$ operate continuously on $A$ as a group of automorphisms. If $g \in G, a \in A$, we denote the image of $a$ under $g$ as $a^{g}$. A map $\theta: G \rightarrow A$ is called a cocycle if $\theta(g h)=\theta(g)^{h} \theta(h)$. Two cocycles are cohomologous if there exists $a \in A$ such that $\theta^{\prime}(h)=a^{h} \theta(h) a^{-1}$ for all $h$. We denote by $H^{1}(G, A)$ the set of cohomology classes.

We consider now two specific calculations of these cohomology sets. Suppose $G=Z_{2}$ and $A=\Gamma_{n}$ and $Z_{2}$ acts on $\Gamma_{n}$ by $K^{s}=D^{-1} K D$ where $S$ is the generator of $Z_{2}, K \in \Gamma_{n}$ and $D=\left[\begin{array}{cc}I & 0 \\ 0 & -I\end{array}\right]$. Thus a cocycle is a map $\theta: Z_{2} \rightarrow \Gamma_{n}$ such that $\theta(1)=I$ and $[D \theta(s)]^{2}=I$ and two cocycles are cohomologous if there exists $K \in \Gamma_{n}$ such that $K D \theta(s) K^{-1}=D \theta^{\prime}(s)$. Now if we identify $\theta$ with $\theta(s)$ and notice that every element of $\Lambda_{n}$ is of the form $D M, M \in \Gamma_{n}$, then it is clear that the set of equivalence classes of elements of $T_{n}$ is the same as the set $H^{1}\left(Z_{2}, \Gamma_{n}\right)$.

Also if $P \Gamma_{n}$ and $P \Lambda_{n}$ are $\Gamma_{n}$ and $\Lambda_{n}$ modulo their centers, then Theorems $\mathrm{A}^{\prime}$ and $\mathrm{B}^{\prime}$ give a description of $H^{1}\left(\mathrm{Z}_{2}, P \Gamma_{n}\right)$. Thus let $\mathrm{Z}_{2}$ act on 
$P \Gamma_{n}$ by $K^{s}=C^{-1} K C$ where $s$ is as above, $K \in P \Gamma_{n}$ and $C$ is the class of $D$, defined above. By the same reasoning used before, we see that $H^{1}\left(Z_{2}, P \Gamma_{n}\right)$ may be identified with the set $S=\left\{L \in P \Lambda_{n} \mid L^{2}=I\right\}$ under the equivalence of conjugation by elements of $P \Gamma_{n}$. Now we have the map $S_{n} \cup T_{n} \rightarrow S$. Notice that two elements of $S_{n} \cup T_{n}$ are equivalent iff their images in $S$ are equivalent. Thus Theorems $\mathrm{A}^{\prime}$ and $\mathrm{B}^{\prime}$ describe $H^{1}\left(\mathrm{Z}_{2}, P \Gamma_{n}\right)$.

2 Proofs of main theorems. We begin with a preliminary result.

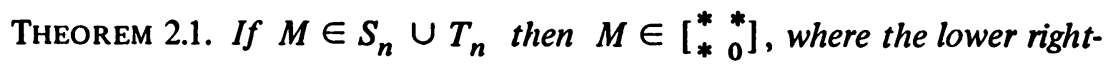
hand $n \times n$ block has nothing but zeros.

Proof. Case 1: $M \in S_{n}$. In this case the proof follows after a sequence of steps.

Step 1. If $C$ is an $n \times n$ skew-symmetric matrix of rank $r$ where $0<$ $r<n$ then there exists a unimodular matrix $U$ such that

$$
U C^{t} U=\left[\begin{array}{ll}
C_{1} & 0 \\
0 & 0
\end{array}\right],
$$

where $C_{1}$ is $r \times r$ and nonsingular.

The proof of this is completely elementary so we omit it.

Step 2. $M$ is equivalent to a matrix whose lower right-hand $n \times n$ block has a right-hand column and a bottom row consisting entirely of zeros.

Proof. Let

$$
M=\left[\begin{array}{cc}
B & A \\
-{ }^{t} A & C
\end{array}\right]
$$

and let

$$
K=\left[\begin{array}{ll}
q_{1} & r_{1} \\
s_{1} & t_{1}
\end{array}\right]+\cdots+\left[\begin{array}{ll}
q_{n-1} & r_{n-1} \\
s_{n-1} & t_{n-1}
\end{array}\right]+I_{2}
$$

where we choose $q_{i}, r_{i}, s_{i}, t_{i}$ as follows. First we find relatively prime $s_{i}$ and $t_{i}$ such that $s_{i} a_{i n}+t_{i} c_{i n}=0$ and then we let $q_{i}$ and $r_{i}$ be such that $q_{i} t_{i}-$ $r_{i} s_{i}=1$. Clearly $K \in \Gamma_{n}$ and a direct claculation shows that $K M^{t} K$ has the desired form.

Step 3. Either $M$ satisfies the conclusion of Theorem 2.1 or $M$ is equivalent to a matrix of the form 


$$
\left[\begin{array}{cc|cc}
B_{1} & B_{2} & A_{1} & 0 \\
-{ }^{t} B_{2} & B_{3} & A_{2} & A_{3} \\
\hline-{ }^{t} A_{1} & -{ }^{t} A_{2} & C_{1} & 0 \\
0 & -{ }^{t} A_{3} & 0 & 0
\end{array}\right],
$$

where $A_{1}, B_{1}, C_{1}$ are $r \times r . A_{3}$ and $B_{3}$ are $n-r \times n-r, B_{2},{ }^{t} A_{2}$ are $r \times n-r$. Also $0<r<n$ and $C_{1}$ is nonsingular.

Proof. By Step 2 we may assume that $r=\operatorname{rank} C<n$. If $r=0$ we are through and if $r>0$ then we apply a matrix $H(U)$ for suitable $U$ to obtain a matrix whose lower right-hand $n \times n$ matrix block has the above form. To finish we note that the facts that $C^{t} A$ is symmetric and $C_{1}$ is nonsingular imply that the upper right $r \times n-r$ block is zero.

We are now ready to give a proof of the theorem in Case 1. Observe that $S_{1}$ is empty and the theorem is true for $S_{2}$ by Step 2 . We proceed by induction. We may assume that $M$ has the form given in Step 3. It is easy to verify that

$$
M_{1}=\left[\begin{array}{cc}
B_{1} & A_{1} \\
-{ }^{t} A_{1} & C_{1}
\end{array}\right] \in S_{r}
$$

and by the induction hypothesis there exists $K_{1}$ such that $K_{1} M_{1}{ }^{t} K_{1}$ has a lower right-hand $r \times r$ block consisting of zeros. Now if we apply $K=K_{1} \dot{ }$ $I_{n-r}$ to $M$ we are through.

Case 2: $M \in T_{n}$. The matrix $T=J M$ has order 2 and therefore $Q^{2 n}=$ $V_{1} \oplus V_{2}$, where $T=+I$ on $V_{1}$ and $T=-I$ on $V_{2}$. If we consider the bilinear form $x \cdot M y$ then we see that $x \cdot M y=-x \cdot J T y= \pm x \cdot J y$, where the last inequality holds when $y \in V_{1} \cup V_{2}$. It now follows from the fact that $M$ is symmetric and $J$ is skew-symmetric that the bilinear forms $x \cdot M y$ and $x \cdot J y$ both vanish if $x, y \in V_{1}$ or $x, y \in V_{2}$. Also the fact that $J\left(V_{i}\right)$ is orthogonal to $V_{i}$ with respect to the usual inner product on $\mathbf{Q}^{2 n}$ implies that $\operatorname{dim} V_{1}=\operatorname{dim} V_{2}=n$. Now if $L_{1}=V_{1} \cap \mathrm{Z}^{2 n}$ then clearly $\operatorname{dim} L_{1}=n$. It is easy to see that $Z^{2 n} / L_{1}$ has no torsion so that there exists an $L_{0}$ such that $Z^{2 n}=L_{0} \oplus L_{1}$. The bilinear forms both vanish and thus there is a unimodular integral matrix $K$ such that $K M^{t} K$ and $K J^{t} K$ both have zeros in the lower $n \times n$ block. It is easy now to find a unimodular integer $S$, with zeros in the upper right-hand $n \times n$ block, such that $S K J^{t} K^{t} S=J$. Hence if $L=S K$, then $L \in \Gamma_{n}$ and $L M^{t} L$ has the desired form. 
COROLlary 2.2. $S_{n}$ is empty if $n$ is odd.

Proof. We know that

$$
M \sim\left[\begin{array}{cc}
B & A \\
-{ }^{t} A & 0
\end{array}\right] \text { and } A^{2}=-I .
$$

Therefore (by taking determinants) $n$ must be even.

Proof of Theorem A By the previous theorem we have that

$$
M \sim\left[\begin{array}{cc}
B & A \\
-A^{t} & 0
\end{array}\right] \text { with } A^{2}=-I \text {. }
$$

We show first that there exists a unimodular $U$ such that $U A U^{-1}=J_{k}, 2 k=n$. Let $e_{i}$ be the usual basis of $\mathrm{Z}^{n}$ and let $A e_{1}=a_{1} e_{1}+\cdots+a_{n} e_{n}$. Since $A$ is unimodular $\left(a_{1}, \cdots, a_{n}\right)=1$. (This notation indicates g.c.d.) Hence by Dirichlet's theorem there exists an integer $m$ such that $\left(a_{2}+m a_{1}, a_{3}, \cdots, a_{n}\right)$ $=1$. Thus after an elementary row and column operation we may assume $\left(a_{2}\right.$, $\left.\cdots, a_{n}\right)=1$. If $V_{0}$ is the $\mathrm{Z}$-submodule generated by $e_{1}$ and $A e_{1}$ then $\mathrm{Z}^{n} / V_{0}$ has no torsion. To see this assume $w \in V_{0}$ and $w / p \in \mathrm{Z}^{n}-V_{0}$, where $p$ is a prime number. Then $w=\alpha e_{1}+\beta A e_{1}=\left(\alpha+\beta a_{1}\right) e_{1}+\beta a_{2} e_{2}+$ $\cdots+\beta a_{n} e_{n}, \alpha, \beta \in Z$. Since $\left(a_{2}, \cdots, a_{n}\right)=1, p \mid \beta$ and hence $p \mid \alpha$, a contradiction. Thus $V_{0}$ is a direct summand of $Z^{n}$. We may continue in this way and after rearranging the vectors in the new basis we obtain the desired result. Thus after applying $H(U)$ we see that $M$ is equivalent to a matrix of the form $\left[\begin{array}{ll}B & J \\ J & 0\end{array}\right]$. If we write

$$
B=\left[\begin{array}{cc}
B_{1} & B_{2} \\
-{ }^{t} B_{2} & B_{3}
\end{array}\right]
$$

then it follows from the definition of $S_{n}$ that $B_{1}=B_{2}$ and $B_{2}={ }^{t} B_{2}$. It is easy now to find a suitable symmetric matrix $S$ so that after applying $G(S)$ we get the desired form.

Before we prove Theorem B we need some preliminary lemmas and calcula. tions.

LEMma 2.3. Let $M_{i}, N_{i} \in S_{n_{i}}\left(T_{n_{i}}\right)$ with $M_{i} \sim N_{i}$. Then $M_{1}+\cdots \dot{+}$ $M_{n} \sim N_{1}+\cdots \dot{+} N_{n}$.

Proof. If $K_{i} \in \Gamma_{n_{i}}$ is such that $K_{i} M_{i}{ }^{t} K_{i}=N_{i}$ then we need only apply $K_{1}+\cdots+K_{n}$ to $M_{1}+\cdots+M_{n}$ to finish. 
LEMMA 2.4. The group of matrices which permute the coordinates of the points in $R^{n}$ is a subgroup of the unimodular group. In fact if $U$ is such a permutation matrix then ${ }^{t} U=U^{-1}$.

Proof. Trivial.

LEMMA 2.5. If

$$
B=\left[\begin{array}{ll}
B_{1} & B_{2} \\
{ }^{t} B_{2} & B_{3}
\end{array}\right]
$$

is symmetric, with $B_{1}$ unimodular, then

$$
\left[\begin{array}{ll}
B & I \\
I & 0
\end{array}\right] \sim\left[\begin{array}{cc}
B^{\prime} & I \\
I & 0
\end{array}\right] \text {, where } B^{\prime}=\left[\begin{array}{cc}
B_{1} & 0 \\
0 & *
\end{array}\right] \text {. }
$$

Proof. Let

$$
U=\left[\begin{array}{cc}
I & 0 \\
-{ }^{t} B_{2} B_{1} & I
\end{array}\right]
$$

and apply $H(U)$.

LEMMA 2.6. We need several facts which we collect here.

(a)

$$
\left[\begin{array}{cc}
0 & -A \\
-A & 0
\end{array}\right] \sim\left[\begin{array}{ll}
0 & A \\
A & 0
\end{array}\right]
$$

(b) $\quad\left[\begin{array}{ll}B & I_{n} \\ I_{n} & 0\end{array}\right] \sim\left[\begin{array}{rr}B & -I_{n} \\ -I_{n} & 0\end{array}\right]$, if $B$ is symmetric with $B^{2}=I_{n}$,

(c)

$$
\left[\begin{array}{ll}
I_{2} & F_{1} \\
F_{1} & 0
\end{array}\right] \sim\left[\begin{array}{ll}
I_{2} & I_{2} \\
I_{2} & 0
\end{array}\right]
$$

(d)

$$
\left[\begin{array}{ll}
F_{1} & I_{2} \\
I_{2} & 0
\end{array}\right] \sim\left[\begin{array}{ll}
0 & F_{1} \\
F_{1} & 0
\end{array}\right]
$$

(e)

$$
\left[\begin{array}{ll}
1 & 1 \\
1 & 0
\end{array}\right]+\left[\begin{array}{ll}
F_{1} & I_{2} \\
I_{2} & 0
\end{array}\right] \sim\left[\begin{array}{ll}
I_{3} & I_{3} \\
I_{3} & 0
\end{array}\right] .
$$


Proof. In each case we apply an appropriate matrix to the left-hand side. We list matrices below. Each of these matrices belongs to $\Gamma_{n}$ for the appropriate $n$.

(a)

$$
\left[\begin{array}{cc}
0 & -I \\
I & 0
\end{array}\right]
$$

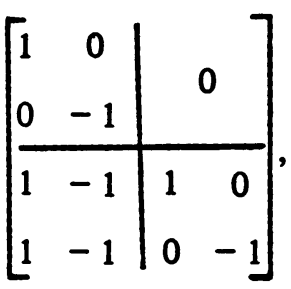

(c)

(b)

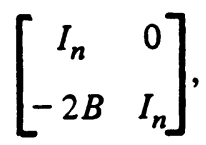

$$
\left[\begin{array}{ccc|ccc}
-1 & 1 & -1 & 0 & 1 & 0 \\
1 & 0 & 1 & -1 & 0 & 1 \\
1 & 1 & 0 & 0 & 0 & -1 \\
\hline & & & -1 & 1 & 1 \\
& 0 & & -1 & 0 & 2 \\
& & & 1 & 0 & -1
\end{array}\right] .
$$

Corollary 2.7. $M(0, t) \sim-M(0, t)$. (We recall that $M(0, t)$ is defined in the statement of Theorem B.)

Proof. This follows by Lemma 2.6(a) and Lemma 2.3.

LEMma 2.8. Given a symmetric matrix $B$, there exist positive integers $k$ and $j$ such that if

$$
M_{1}=\left[\begin{array}{ll}
B & I \\
I & 0
\end{array}\right] \text { and } M_{2}=\left[\begin{array}{cc}
B & -I \\
-I & 0
\end{array}\right], \quad M_{i} \in \Gamma_{n},
$$

then

$$
M_{1} \sim\left[\begin{array}{lll|l}
I_{k} & & & \\
& F_{j} & & I \\
& 0 & \\
\hline & I & 0
\end{array}\right] \text { and } M_{2} \sim\left[\begin{array}{rr|r}
I_{k} & & \\
& F_{j} & -I \\
& &
\end{array}\right] .
$$


Proof. We prove this only for $M_{1}$ since a similar argument may be applied for $M_{2}$. The proof is by induction.

For a suitable symmetric $S, G(S) M^{t} G(S)=\left[\begin{array}{cc}B^{\prime} & I \\ I & 0\end{array}\right]$ has only 0 's and 1 's in the uper left-hand $n \times n$ block. Thus we may assume that each entry of $B$ is either 0 or 1 .

We consider two cases.

Case 1. $b_{i i}=1$ for some $i$. By applying $H(U)$ for a suitable permutation matrix $U$ (Lemma 2.4) we may assume that $i=1$. Now by Lemma 2.5 we may assume all other elements of the first row and column are zero. Now by the induction hypothesis and Lemma 2.3 we obtain the desired result.

Case 2. $b_{i i}=0$, for all $i$. If all the entries in the first row are 0 then by the induction hypothesis, Lemma 2.3 and Lemma 2.4 we get the desired result.

If all the entries in the first row are not 0 , then by applying $H(U)$ for a suitable permutation matrix $U$ (Lemma 2.4) we see that

$$
M_{1} \sim\left[\begin{array}{cc|c|c}
0 & 1 & & \\
1 & 0 & B_{2} & \\
\cline { 1 - 2 }{ }^{t} B_{2} & B_{3} & \\
\hline & & \\
\cline { 1 - 1 } I & & 0
\end{array}\right] .
$$

By Lemma 2.5 we may take $B_{2}=0$. Lemma 2.8 now follows by the induction hypothesis, Lemma 2.3 and Lemma 2.4.

N. B. To get the corresponding result for $M_{2}$ it is crucial to observe that $k$ and $j$ depend only on the parity of the entries of $B$.

Corollary 2.9. $M_{1} \sim M_{2}$.

Proof. By the previous theorem we see that this follows immediately from Lemmas 2.6(a), (b) and 2.3.

LEMMA 2.10. If

$$
M=\left[\begin{array}{cc}
B & F_{n} \\
F_{n} & 0
\end{array}\right] \in \Gamma_{2 n},
$$




$$
M \sim\left[\begin{array}{cc|c}
I_{2 m} & 0 & F_{n} \\
0 & 0 & \\
\hline F_{n} & 0
\end{array}\right] .
$$

Proof. We write $B=\left[B_{i j}\right]$, where $B_{i j}$ is a $2 \times 2$ matrix. The symmetry of $B F_{n}$ implies

$$
B_{i j}=\left[\begin{array}{ll}
a_{i j} & b_{i j} \\
b_{i j} & a_{i j}
\end{array}\right]
$$

And the symmetry of $B$ implies $a_{i j}=a_{i j}$ and $b_{i j}=b_{j i}$. Now we define a matrix $S=\left[S_{i j}\right]$, where $S_{i j}$ is a $2 \times 2$ matrix, by

$$
\begin{aligned}
& S_{i j}=\left[\begin{array}{cc}
-b_{i j} & -a_{i j} \\
0 & 0
\end{array}\right], \quad i<j, \\
& S_{i j}=\left[\begin{array}{rr}
-a_{i j} & 0 \\
-b_{i j} & 0
\end{array}\right], \quad i>j
\end{aligned}
$$

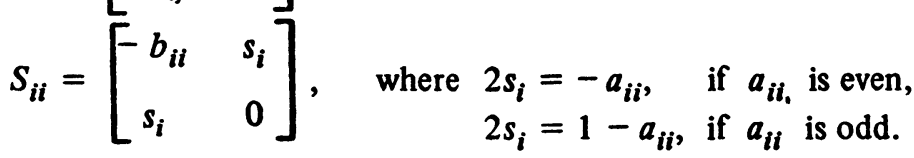

$S$ is symmetric and

$$
G(S) M^{t} G(S)=\left[\begin{array}{c|c}
B+S F_{n}+F_{n} S & F_{n} \\
\hline F_{n} & 0
\end{array}\right] .
$$

$B+S F_{n}+F_{n} S=\left[B_{i j}+S_{i j} F_{1}+F_{1} S_{i j}\right]$. Also, $B_{i j}+S_{i j} F_{1}+F_{1} S_{i j}=0$, if $i \neq j$, and $B_{i i}+S_{i i} F_{1}+F_{1} S_{i i}=I_{2}$ or 0 . Thus, by applying Lemma 2.4, Lemma 2.10 follows.

Proof of Theorem B. If $M \in T_{n}$, then by Theorem 2.1 we may assume $M$ is of the form

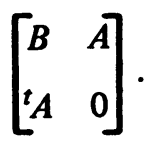


The case $n=1$ is now easy. Thus we consider the case $n>1$. It is a fact, proved in [3], that for any matrix $A$, with $A^{2}=I$, there exists a unimodular $U$ such that

$$
U A U^{-1}=\left[\begin{array}{llll}
I_{r} & & \\
& -I_{s} & \\
& & \\
& & F_{t}
\end{array}\right]
$$

Thus by applying $H(U)$ we see that

$$
M \sim\left[\begin{array}{cc|ccc} 
& & I_{r} & & \\
& B \cdot & & -I_{s} & \\
\hline I_{r} & & & & F_{t} \\
& -I_{s} & & & \\
& & F_{t} & &
\end{array}\right] .
$$

Now if

$$
B=\left[\begin{array}{ll}
* & * \\
* & B_{1}
\end{array}\right]
$$

where $B_{1}$ is $2 t \times 2 t$, then

$$
N=\left[\begin{array}{ll}
B_{1} & F_{t} \\
F_{t} & 0
\end{array}\right] \in \Gamma_{2 t}
$$

and by Lemma 2.10

$$
N \sim\left[\begin{array}{cc|c}
I_{2 m} & 0 & \\
0 & 0 & F_{t} \\
\hline F_{t} & 0
\end{array}\right] .
$$

By Lemma 2.6(c) and (d) and Lemma 2.3 


$$
N \sim\left[\begin{array}{cc|c}
I_{2 m} & & \\
& F_{t-m} & I_{2 t} \\
\hline I_{2 t} & 0
\end{array}\right]=N^{\prime}
$$

If $K \in \Gamma_{2 t}$ is such that $K N^{t} K=N^{\prime}$, then if we first apply $I_{r+s}+K$ to $M$ and then use Lemma 2.4 we see that

$$
M \sim\left[\begin{array}{cc|cc}
* & 0 & I_{r+2 t} & 0 \\
0 & B_{1}^{\prime} & 0 & -I_{s} \\
\hline I_{r+2 t} & 0 & 0 \\
0 & -I_{s} & &
\end{array}\right] .
$$

And by the Corollary 2.9 and Lemma 2.3 this implies that

Now apply Lemma 2.8 and we see

$$
M \sim\left[\begin{array}{cc}
B^{\prime} & I \\
I & 0
\end{array}\right] .
$$

$$
M \sim\left[\begin{array}{ccc|c}
I_{k} & & & \\
& F_{j} & & I \\
& 0 & \\
\hline I & & 0
\end{array}\right] .
$$

But by Lemma $2.6(\mathrm{c})$ we get

$$
M \sim\left[\begin{array}{ll}
I_{k} & I_{k} \\
I_{k} & 0
\end{array}\right]+\left[\begin{array}{cc}
0 & F_{j} \\
F_{j} & 0
\end{array}\right]+\left[\begin{array}{cc}
0 & I_{w} \\
I_{w} & 0
\end{array}\right] .
$$

Now if $k \geqslant 3$, by repeated use of Lemma 2.6(e) we see that

$$
\left[\begin{array}{ll}
I_{k} & I_{k} \\
I_{k} & 0
\end{array}\right] \sim\left[\begin{array}{ll|ll}
I_{r} & 0 & I_{u} & \\
0 & 0 & & F_{v} \\
\hline I_{u} & & 0 \\
& F_{v} &
\end{array}\right], \quad r=0,1, \text { or } 2 .
$$


Thus, by Lemma 2.3 and Lemma 2.4, $M \sim M(r, v+j)$.

To finish the proof it remains to show that no two distinct matrices of the form $M(r, t)$ are equivalent. Now if we define

$$
N(r, t)=\left[\begin{array}{ccc|c}
I_{r} & & & \\
& 0 & & I \\
& F_{t} & \\
\hline I & & 0
\end{array}\right],
$$

then, by Lemma 2.6(d) and Lemma 2.3, $M(r, t) \sim N(r, t)$. Thus the problem is reduced to showing that $N(r, t) \not N(u, v)$ if $r \neq u$ or $t \neq v$. So suppose there exists a $K \in \Gamma_{n}, K=\left[\underset{S}{Q}{ }_{T}^{R}\right]$, such that $K N(r, t)^{t} K=N(u, v)$, or equivalently, $K N(r, t)=N(u, v)^{t} K^{-1}$. After multiplying, it-is clear that we must have $S=0$ as well as the following:

$$
\begin{aligned}
Q^{t} T & =T^{t} Q=I_{n}, \\
2 R & =\left[\begin{array}{lll}
I_{u} & & \\
& 0 & \\
& & F_{v}
\end{array}\right] T-Q\left[\begin{array}{lll}
I_{r} & & \\
& 0 & \\
& & F_{t}
\end{array}\right] .
\end{aligned}
$$

The natural map $\mathbf{Z} \rightarrow \mathrm{Z}_{2}$ induces a ring homomorphism from $\mathrm{GL}(n, \mathrm{Z})$ onto $\mathrm{GL}\left(n, \mathbf{Z}_{2}\right)$. If $a \in \mathbf{Z}, A \in \mathrm{GL}(n, \mathbf{Z})$, denote their images under this map by $a^{\prime}, A^{\prime}$ respectively. However, without confusion, we denote the images of $1,0, I_{j}, F_{j}$ by $1,0, I_{j}, F_{j}$. Thus (1) and (2) imply

$$
\begin{aligned}
Q^{\prime} t^{\prime} & =T^{\prime t} Q^{\prime}=I_{n}, \\
0 & =\left[\begin{array}{lll}
I_{u} & & \\
& 0 & \\
& & \\
& & F_{v}
\end{array}\right] T^{\prime}-Q^{\prime}\left[\begin{array}{lll}
I_{r} & & \\
& 0 & \\
& & \\
& & F_{t}
\end{array}\right] .
\end{aligned}
$$

Thus

$$
\left[\begin{array}{lll}
I_{u} & & \\
& 0 & \\
& & F_{v}
\end{array}\right]=Q^{\prime}\left[\begin{array}{lll}
I_{r} & & \\
& 0 & \\
& & F_{t}
\end{array}{ }^{t} Q^{\prime}\right.
$$


And by comparing the ranks of these two matrices we see that $u-r=$ 2(v-t). Thus if $u$ and $r$ are not equal we need only consider the case $u=2$, $r=0$. Now from $\left(2^{\prime}\right)$ we see that $[0, \cdots, 0]=\left[t_{11}^{\prime}, t_{12}^{\prime}, \cdots, t_{1 n}^{\prime}\right]-\left[0, \cdots, q_{1 s+2}^{\prime}, q_{1 s+1}^{\prime}, q_{1 s+4}^{\prime}, q_{1 s+3}^{\prime}, \cdots\right]$, where $s=n-2 t$. Thus $t_{i j}^{\prime}=0$ for $j=1, \cdots, s$ and $t_{1 j}^{\prime}=q_{1 j+1}^{i}, j=s+1, s+3, \cdots, \quad t_{1 j}^{\prime}=q_{1 j-1}^{\prime}, j=s+2, s+4, \cdots$.

Thus $\Sigma_{j=1}^{s} t_{1 j}^{\prime} q_{1 j}^{\prime}=0$ and $t_{1 j}^{\prime} q_{1 j}^{\prime}+t_{1 j+1}^{\prime} q_{1 j+1}^{\prime}=0$ for $j=s+1, s+3$, $\cdots$ and therefore $\sum_{j=1}^{n} t_{1 j}^{\prime} q_{1 j}^{\prime}=0$. But $\left(1^{\prime}\right)$ implies that $\sum_{j=1}^{n} t_{1 j}^{\prime} q_{1 j}^{\prime}=1$, a contradiction. So we must have $u=r$ and hence also $v=t$. Q.E.D.

Proof of Corollary 1.3. We prove equivalently that if $M \in T_{n} \cup S_{n}$ then $M \sim-M$. If $M \in S_{n}$ this is trivial. If $M \in T_{n}$ then $M \sim M(r, t)$. Now $M(r, t)=M_{1}+M_{2}$, where

$$
M_{1}=\left[\begin{array}{cc}
I_{s} & I_{s} \\
I_{s} & 0
\end{array}\right] \text { and } M_{2}=\left[\begin{array}{c|c|cc}
0 & I_{r-s} & \\
& & F_{t} \\
\hline I_{r-s} & & 0 \\
& F_{t} &
\end{array}\right]
$$

Now

$$
\begin{array}{rlrl}
M_{1} & \sim\left[\begin{array}{cc}
I_{s} & -I_{s} \\
-I_{s} & 0
\end{array}\right] \quad \text { by Lemma 2.6(b), } \\
& \sim-M \quad & \text { by applying } G\left(I_{s}\right) .
\end{array}
$$

Also $M_{2} \sim-M_{2}$ by Lemma 2.6(a). Hence, by Lemma 2.3, $M(r, t) \sim$ $-M(r, t)$.

3. Symmetric and pseudo-symmetric Riemann surfaces. In this section we consider the problem mentioned in Remark 1.2. For future reference we define the following matrix.

$$
W=\left[\begin{array}{cc}
J_{k} & 0 \\
0 & -J_{k}
\end{array}\right]
$$

Clearly $W \in S_{n}, n=2 k$.

Let $X$ be a Riemann surface of genus $n$ and let $\Sigma$ denote a canonical homology basis for $X$. That is, $\Sigma=\left(A_{1}, \cdots, A_{n}, B_{1}, \cdots, B_{n}\right)$, a collection 
of $2 n$ homology classes of loops with $A_{i} \times A_{j}=B_{i} \times B_{j}=0, A_{i} \times B_{j}=\delta_{i j}$. Here " $x$ " denotes intersection number. If $f$ is any automorphism of $X$ then let $\varphi(f, \Sigma)$ denote the matrix of the induced action on $H_{1}(X)$ with respect to $\Sigma$. It is well known that $\varphi(f, \Sigma) \in \Gamma_{n}$ (resp. $\Lambda_{n}-\Gamma_{n}$ ) if $f$ is orientation preserving (resp. reversing). If $\psi_{1}, \cdots, \psi_{n}$ is a basis for the space of holomorphic 1 -forms on $X$ then the matrix $Z=\left[\int_{B_{i}} \psi_{j}\right]\left[\int_{A_{i}} \psi_{j}\right]^{-1}$ is called a period matrix of $X$. It may be shown that $Z$ is independent of the choice of $\psi_{1}$, $\cdots, \psi_{n}$ and $Z \in H_{n}$, the Siegel upper half-plane. Also there is an action of $\Lambda_{n}$ on $H_{n}$ given as follows. Let $M=\left[\begin{array}{ll}A & B \\ C & D\end{array}\right] \Lambda_{n}$; then

$$
\begin{aligned}
Z \cdot M & =(D Z+B)(C Z+A)^{-1} \quad \text { if } M \in \Lambda_{n}, \\
& =(D \bar{Z}+B)(C \bar{Z}+A)^{-1} \quad \text { if } M \in \Lambda_{n}-\Gamma_{n} .
\end{aligned}
$$

LEMMA 3.1. If $f$ is a holomorphic or conjugate holomorphic automorphism of $X$, then $Z \cdot \varphi(f, \Sigma)=Z$.

Proof. Given any basis $\psi_{1}, \cdots, \psi_{n}$ of the holomorphic 1 -forms it is true that $f^{*} \psi_{1}, \cdots, f^{*} \psi_{n}$ is also a basis if $f$ is holomorphic. If $f$ is conjugate holomorphic then $\overline{f^{*} \psi_{1}}, \cdots, \overline{f^{*} \psi_{n}}$ is a basis. Here $f^{*} \psi_{i}$ denotes pullback of $\psi_{i}$ by $f$. Now if we compute the period matrix of $X$ with respect to these two sets of bases and set them equal we get our result.

DEFinition 3.2. A reflection (resp. a pseudo-reflection) $f$ of a Riemann surface $X$ is a conjugate holomorphic automorphism $f$ such that $f^{2}=$ id (resp. $f^{2}$ induces - id on $H_{1}(X)$ ).

TheOREM 3.3. Suppose $X$ is a symmetric (resp. pseudo-symmetric) Riemann surface with a reflection $f$ (resp. pseudo-reflection). Then it is possible to choose a canonical homology basis $\Sigma$ such that $\varphi(f, \Sigma)=Y(r, t)$ (resp. $\varphi(f, \Sigma)=W$ ). Hence $Z \cdot Y(r, t)=Z$ (resp. $Z \cdot W=Z$ ). Conversely, if $X$ is a Riemann surface with a period matrix $Z$ such that $Z \cdot Y(r, t)=Z($ resp. $Z \cdot W=Z)$ then there exists a canonical homology basis $\Sigma$ and a reflection (resp. a pseudoreflection) $f$ such that $\varphi(f, \Sigma)=Y(r, t)($ resp. $\varphi(f, \Sigma)=W)$.

Proof. We consider the case when $X$ is symmetric. Pick any canonical homology basis $\Sigma^{\prime}$, and let $M=\varphi\left(f, \Sigma^{\prime}\right)$. Clearly $M \in T_{n}$. Then by Theorem $\mathrm{B}^{\prime}$ there is a matrix $K \in \Gamma_{n}$ such that $K M K^{-1}=Y(r, t)$ for a unique $Y(r, t)$. If we apply the matrix $K$ to the homology classes of loops in $\Sigma^{\prime}$ then we obtain a new canonical homology basis $\Sigma$ such that $(f, \Sigma)=Y(r, t)$. A similar argument holds in the pseudo-symmetric case (here we use Theorem $\mathrm{A}^{\prime}$ ).

To obtain the converse we use Torelli's theorem. Thus if $Z \cdot M=Z$ then $X$ has a conjugate holomorphic map $f$ and a canonical homology basis $\Sigma^{\prime}$ such 
that $\varphi\left(f, \Sigma^{\prime}\right)=M$ or $-M$. But by Corollary 1.3 there exists a $K \in \Gamma_{n}$ such that $K M K^{-1}=-M$ and hence for a suitable $\Sigma, \varphi(f, \Sigma)=M$.

REMARK 3.4. It follows by Theorem $\mathrm{A}^{\prime}$ that $S_{n}$ is empty if $n$ is odd. Hence there exist no pseudo-symmetric Riemann surfaces of odd genus. The existence of pseudo-symmetric Riemann surfaces of every even genus was established by Singerman [4].

THEOREM 3.5. If $M \in T_{n}$ (resp. $S_{n}$ ) then there exist a Riemann surface $X$ of genus $n$, a canonical homology basis $\Sigma$, and a reflection $f$ (resp. a pseudo-reflection $f$ ) such that $\varphi(f, \Sigma)=M$.

Proof. We consider the case $M \in T_{n}$ first. Let $X$ be a smooth compact oriented surface of genus $n$ as shown in Figure 1. We show there exists

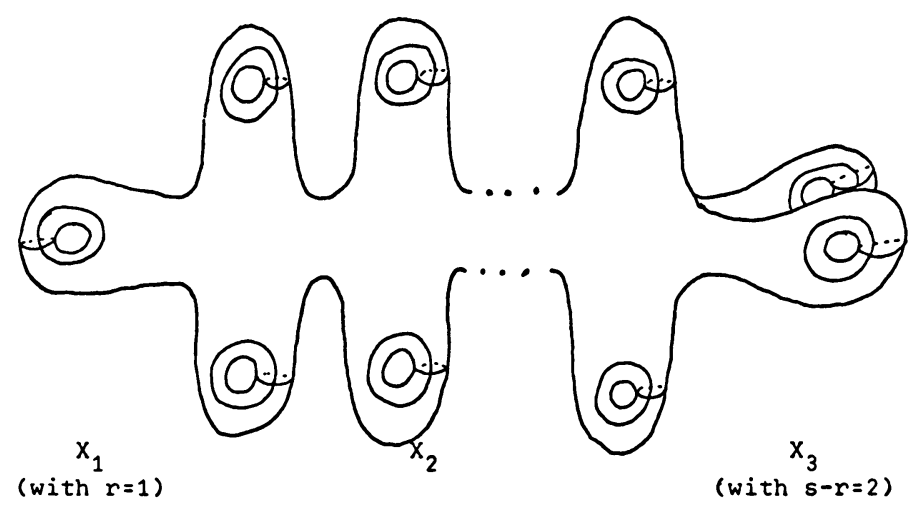

FiguRe 1

an orientation reversing (smooth) automorphism $f$ of order 2 which induces $Y(r, t)$ on $H_{1}(X)$. First we dissect $X$ into three parts $X_{1}, X_{2}, X_{3}$ and define $f$ on each of these parts separately. We note $X_{1}$ consists of $r$ handles $(r=0$, $1,2), X_{2}$ consists of $2 t$ handles and $X_{3}$ consists of $s-r$ handles. On $X_{2}$ and $X_{3}, f$ is just a vertical reflection. In order to define $f$ on $X_{1}$ we first remark that a torus has an orientation reversing map of order 2 , call it $g$, whose fixed point set consists of exactly one Jordan curve. The quotient surface obtained is a Moebius strip and, for a suitably chosen canonical homology base, the induced action on $H_{1}(X)$ has the matrix $\left[\begin{array}{ll}1 & 1 \\ 1 & 0\end{array}\right]$. To see this we think of the torus as the quotient of the plane by the lattice generated by $1, \tau$, where $\operatorname{Im}(\tau)>0, \operatorname{Re}(\tau)$ $=1 / 2$. The map $z \rightarrow \bar{z}$ covers $g$. Now remove a disk which intersects the fixed 
point set of $g$ and which $g$ maps onto itself. Attach $r=0,1,2$ copies of the resulting surface to obtain $X_{1}$ and let $f$ be the restriction of $g$. We may now put a conformal structure on $X$ so that $f$ is conjugate holomorphic. If $\Sigma^{\prime}$ consists of two loops around each handle as shown then clearly $\varphi\left(f, \Sigma^{\prime}\right)=$ $Y(r, t)$. Now, given any $M \in \Lambda_{n}-\Gamma_{n}$ of order 2 , by Theorem $\mathrm{B}^{\prime}$ there exists $K \in \Gamma_{n}$ such that $K M K^{-1}=Y(r, t)$. Hence for an appropriate choice of canonical homology base $\Sigma, \varphi(f, \Sigma)=M$.

The case $M \in S_{n}$ is simpler. As was previously remarked we know that $n$ must be even and that there exists a pseudo-symmetric surface of genus $n$. Let $X$ be such a surface with pseudo-reflection $f$ and let $\Sigma^{\prime}$ be a canonical homology base. Then $\varphi\left(f, \Sigma^{\prime}\right) \in S_{n}$ and by Theorem $\mathrm{A}^{\prime}$ there exists a $K \in \Gamma_{n}$ such that $M=K \varphi\left(f, \Sigma^{\prime}\right) K^{-1}$. Thus for a suitable canonical homology base $\Sigma$, $\varphi(f, \Sigma)=M$.

It is now an easy matter to obtain the relationship between the topological type of the quotient surface $X / f$ and the equivalence class of $\varphi(f, \Sigma)$ in $S_{n}$ or $T_{n}$.

THEOREM 3.6. (1) Let $X$ be symmetric with reflection $f$. Then

(a) $X / f$ is a nonorientable surface with $2 t+r$ cross-caps and $s-$ $r+1$ boundary curves iff $\varphi(f, \Sigma)=Y(r, t)$ for suitable $\Sigma$, and $r>0$.

(b) $X / f$ is an orientable surface with $t$ handles and $s+1>2$ boundary curves iff $\varphi(f, \Sigma)=Y(0, t)$, for suitable $\Sigma$.

(c) Assume $s=0$ or 1 . Then $X / f$ is either an orientable surface with $t$ handles and $s+1$ boundary curves or a nonorientable surface with $s+1$ cross-caps iff $\varphi(f, \Sigma)=Y(0, t)$ for suitable $\Sigma$.

(2) If $X$ is pseudo-symmetric with pseudo-reflection $f$ then $X / f$ is the projective plane.

Proof. Let $X / f$ have the topological type of either (1)(a) or (b). In (1)(a) we may assume that $X / f$ has $r$ cross-caps, $t$ handles and $s-r+1$ boundary curves (see e.g. [1, p. 98]). By the proof of Theorem 2 there exist a smooth compact oriented surface $X^{\prime}$, an orientation reversing diffeomorphism $f^{\prime}$ and a canonical homology base $\Sigma^{\prime}$ such that $X^{\prime} / f^{\prime}$ has the same topological type as $X / f$ and $\varphi\left(f^{\prime}, \Sigma^{\prime}\right)$ has the desired form. Now let $h: X^{\prime} / f^{\prime} \rightarrow$ $X / f$ be a diffeomorphism. We may put a Klein surface structure on $X^{\prime} / f^{\prime}$ so that $h$ is dianalytic (see [2] for definitions). Also we can put a Riemann surface structure on $X^{\prime}$ so that $f^{\prime}$ is a reflection and the projection $X^{\prime} \rightarrow X^{\prime} / f^{\prime}$ is dianalytic. It follows now by 1.9.3 of [2] that we may lift $h$ to a biholomorphic map $h^{\prime}: X^{\prime} \rightarrow X$. Now let $\Sigma=h^{\prime}\left(\Sigma^{\prime}\right)$ and $\varphi(f, \Sigma)$ has the desired form. This shows necessity in (1)(a) and (b). 


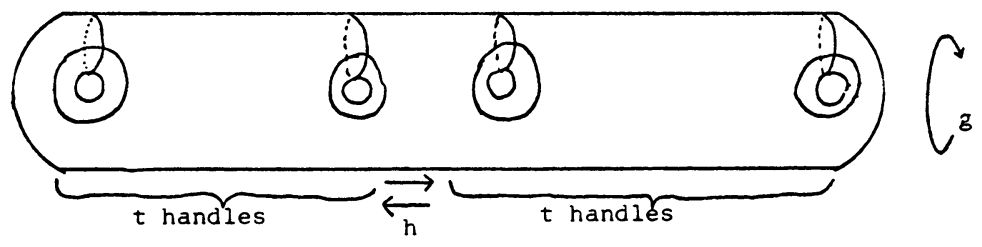

$(\propto)$

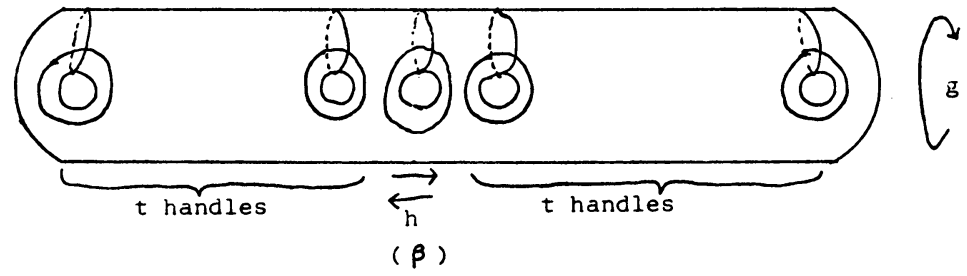

FIGURE 2

To show necessity in (1)(c) we consider $X$ and $\Sigma$ to be as shown in Figure 2, where $(\alpha)$ and $(\beta)$ represent the cases $r=0$ and $r=1$ respectively. If $g$ is a rotation and $h$ is left-right reflection as shown, then $\varphi\left(g h, \Sigma^{\prime}\right)=-Y(0, t)$, $\varphi\left(h, \Sigma^{\prime}\right)=Y(0, t)$. But by Corollary $1.3, \varphi(g h, \Sigma)=Y(0, t)$ for suitable $\Sigma$. Now we proceed as in (1)(a) and (b) to finish.

To show sufficiency in (1)(a), (b), (c) we first remark that it follows from the discussion in [2] that for any surface $X$ with reflection $f, X / f$ is never an orientable surface without boundary and that the set of all $X / f$ 's consists precisely of the set of all other compact surfaces $Y$ which satisfy $\chi(X)=2 \chi(Y)$, where " $\chi$ " is the Euler characteristic. By checking all the cases we see that $X / f$ must have the topological type described in either (1)(a), (b) or (c). To finish use the fact that the matrices $Y(r, t)$ are inequivalent in $T_{n}$ (Theorem $\mathrm{B}^{\prime}$ ).

To prove (2) we observe that $f$ induces a reflection $f^{\prime}$ on $X / f^{2}$. Since $X / f^{2}$ is a sphere, $f^{\prime}$ is either a vertical reflection or the antipodal map [2, 1.9.4]. We may eliminate the first case since if $f^{\prime}$ had a fixed curve then so would $f$ and hence so would $f^{2}$.

REMARK 3.7. We should mention that (1)(a), (b) and (c) of the previous theorem yield an alternative proof of part of Theorem 3.3.

\section{REFERENCES}

1. L. V. Ahlfors and L. Sario, Riemann surfaces, Princeton Math. Ser., no. 26, Princeton Univ. Press, Princeton, N. J., 1960. MR 22 \#5729.

2. N. L. Alling and N. Greenleaf, Foundations of the theory of Klein surfaces, Lecture 
Notes in Math., vol. 219, Springer-Verlag, New York, 1971.

3. I. Reiner, Integral representations of cyclic groups of prime order, Proc. Amer. Math. Soc. 8 (1957), 142-146. MR 18,717.

4. D. Singerman, Riemann surfaces which are conformally equivalent to their conjugates (preprint).

5. T. A. Springer, Galois cohomology of linear algebraic groups, Algebraic Groups and Discontinuous Subgroups (Proc. Sympos. Pure Math., Boulder, Colo., 1965), Amer. Math. Soc., Providence, R. I., 1966, pp. 149-158. MR 35 \#1600.

DEPARTMENT OF MATHEMATICS, NORTHERN ILLINOIS UNIVERSITY, DEKALB, ILLINOIS 60115 (Current address)

INSTITUT FÜR MATHEMATIK, RUHR-UNIVERSITÄT BOCHUM, 4630 BOCHUM, FEDERAL REPUBLIC OF GERMANY 